\title{
NUMERICAL RANGES OF TENSOR PRODUCTS OF OPERATORS
}

\author{
TEISHIRÔ SAITÔ \\ (Received November 16, 1966)
}

1. In a recent paper [1], A. Brown and C. Pearcy proved that if $A$ and $B$ are bounded linear operators on a Hilbert space, then $\sigma(A \otimes B)=\sigma(A) \cdot \sigma(B)$, where $\sigma(\cdot)$ denotes the spectrum of an operator. In connection with this theorem, we shall discuss an analogous relation among the numerical ranges of $A, B$ and $A \otimes B$.

2. In the sequel, spaces under consideration will be complex Hilbert spaces, and all operators will be assumed to be bounded and linear. The spectrum of any operator $T$ is denoted, as above, by $\sigma(T)$ and the numerical range of $T$ is denoted by $W(T)$. For an arbitrary set $S$ in the complex plane, $\operatorname{co}(S)$ means the convex hull of $S$ and we write $\bar{S}$ for the closure of $S$. It is known that $\operatorname{co}(\sigma(T))$ is closed. Motivated by the above theorem by A. Brown and C. Pearcy, the following questions are naturally raised.

(I) Does the following relation (*) hold for any operators $A$ and $B$ ?

$$
\overline{W(A \otimes B})=\overline{\operatorname{co}}(W(A) \cdot W(B)) .
$$

(II) If the relation (*) is not always true, when does the relation (*) hold?

3. In this section we shall prove the following two propositions.

Proposition 1. Let $A$ and $B$ be operators on Hilbert space $H$. Then the condition $\bar{W} \overline{(A \otimes B)}=\operatorname{co}(\sigma(A \otimes B))$ implies the relation (*).

Proposition 2. There exist operators $A$ and $B$ on some Hilbert space such as $\overline{W(A \otimes B)} \supsetneq \overline{\mathrm{co}}(W(A) \cdot W(B))$.

To prove Proposition 1, we shall show the following lemma.

LEMMA 1. For arbitrary operators $A$ and $B$ on a Hilbert space $H$ 


$$
\overline{W(A \otimes B}) \supseteqq \overline{\operatorname{co}}(W(A) \cdot W(B)) .
$$

ProOF. If $\lambda \in W(A)$ and $\mu \in W(B)$, there exist unit vectors $x, y \in H$ such as $\lambda=(A x, x)$ and $\mu=(B y, y)$. Thus we have

$$
((A \otimes B) x \otimes y, x \otimes y)=(A x, x)(B y, y)=\lambda \mu
$$

and so $W(A \otimes B) \supseteqq W(A) \cdot W(B)$. Since $\overline{W(A \otimes B)}$ is convex and closed, the conclusion of the lemma is clear.

Proof of Proposition 1. By the theorem of A. Brown and C. Pearcy

$$
\operatorname{co}(\sigma \cdot(A \otimes B))=\operatorname{co}(\sigma \cdot(\mathrm{A}) \cdot \sigma \cdot(B)) \leqq \operatorname{co}(\operatorname{co}(\sigma(A)) \cdot \operatorname{co}(\sigma(B)))
$$

On the other hand, we have

$$
\operatorname{co}(\sigma \cdot(A)) \cdot \operatorname{co}(\sigma(B))) \leqq \overline{\operatorname{co}}(\overline{W(A)} \cdot \overline{W(B)})=\overline{\operatorname{co}}(W(A) \cdot W(B))
$$

since $\operatorname{co}(\sigma(T)) \leqq \overline{W(T)}$ for any operator $T$. Hence, by Lemma 1 and the hypothesis of the proposition, the relation (*) holds.

As a consequence of Proposition 1, we obtain the following result.

COROLLARY. If both $A$ and $B$ are hyponormal operators ${ }^{(*)}$, the relation (*) holds.

ProOF. Since $A^{*} A-A A^{*} \geqq 0$ and $B^{*} B-B B^{*} \geqq 0$, we have

$$
\begin{aligned}
&(A \otimes B)^{*}(A \otimes B)-(A \otimes B)(A \otimes B)^{*} \\
& \quad=\left(A^{*} A-A A^{*}\right) \otimes B^{*} B+A A^{*} \otimes\left(B^{*} B-B B^{*}\right) \geqq 0 .
\end{aligned}
$$

Thus $A \otimes B$ is also a hyponormal operator and so $A \otimes B$ satisfies the condition $\overline{W(A \otimes B})=\operatorname{co}(\sigma(A \otimes B))$ by [2: Corollary 1]. Hence the assertion follows from Proposition 1.

To prove Proposition 2, we shall construct an example. Let $A$ be an operator on a two-dimensional Hilbert space $H$ with the matrix representation $\left(\begin{array}{ll}0 & 1 \\ 0 & 0\end{array}\right)$ and let $B=A^{*}$. Then it is easy to show the following lemma and we omit the proof.

(*) An operator $T$ on a Hilbert space is called to be hyponormal if $T^{*} T \geqq T T^{*}$. 
LEMMA 2. $W(A)=W(B)=\{\lambda:|\lambda| \leqq 1 / 2\}$.

From Lemma 2 we obtain

LEMMA 3. $\overline{\operatorname{co}}(W(A) \cdot W(B)) \subseteq\{\lambda:|\lambda| \leqq 1 / 4\}$.

Proof of Proposition 2. By Lemma 3 it is sufficient to show that $1 / 2 \in W(A \otimes B)$. Since the matrix corresponding to $B$ is $\left(\begin{array}{ll}0 & 0 \\ 1 & 0\end{array}\right)$, the matrix representation of $A \otimes B$ is $\left(\begin{array}{ll}0 & 0 \\ A & 0\end{array}\right)$. For a unit vector $\Phi=\left(\begin{array}{l}x \\ y\end{array}\right) \in H \otimes H$, $((A \otimes B) \Phi, \Phi)=(A x, y)$. Furthermore, for $x=\left(\begin{array}{c}0 \\ 1 / \sqrt{2}\end{array}\right)$ and $y=\left(\begin{array}{c}1 / \sqrt{2} \\ 0\end{array}\right)$ $\in H$, we have $(A x, y)=1 / 2$. Hence $1 / 2 \in W(A \otimes B)$ and the proof is completed.

ADDENDUM. Under the conditions $\overline{W(A)}=\operatorname{co}(\sigma(A))$ and $\overline{W(B)}=\operatorname{co}(\sigma(B))$, the relation (*) is equivalent to the relation $\overline{W(A \otimes B})=\operatorname{co}(\sigma(A \otimes B)$ which is not always insured by the conditions $\overline{W(A)}=\operatorname{co}(\sigma(A))$ and $\overline{W(B)}=\operatorname{co}(\sigma(B))$. In fact, let $X$ be the operator $\left(\begin{array}{ll}0 & 1 \\ 0 & 0\end{array}\right)$ as in the proof of Proposition 2 and $Y$ an operator such as $\sigma(Y)=\overline{W(Y)}=\{\lambda ;|\lambda| \leqq 1 / 2\}$, then we have $\overline{W(S \otimes T})$ Fo $(\sigma(S \otimes T))$ where $S=X \oplus Y$ and $T=S^{*}$, while $\overline{W(S)}=\operatorname{co}(\sigma(S))=\{\lambda:|\lambda| \leqq 1 / 2\}$ and $\overline{W(T)}=\operatorname{co}(\sigma(T))=\{\lambda:|\lambda| \leqq 1 / 2\}$.

\section{REFERENCES}

[1] A. BRown AND C. PEARCy, Spectra of tensor products of operators, Proc. Amer. Math. Soc., 17(1966), 162-166.

[2] T. SAITô AND T. Yoshino, On a conjecture of Berberian, Tôhoku Math. Journ., 17 (1965), 147-149.

The Collge of General Education TÔHOKU UNIVERSITY, SENDAI, JAPAN. 